souterraines. Il est intéressant de rapprocher cette station accidentelle des habitudes normalement nivicoles de ces insectes.

D'autre part, M. P. DE Pfyerimhoff signale la découverte, dans le Sud de la Tunisie, de Sefrania Bleusei Pic, [Col. Dermestidae] que représente la vignette de ce Bulletin (cf. supra, p. 9). Un individu de grande taille $(4,2$ mill.), trouvé à Nefta contre les vitres d'une fenètre, lui a été donné par M. F. Pectier.

La capture répétée de ce Dermestide sur les fenêtres des appartements est à remarquer; mais il semble peu probable qu'il faille y voir un insecte nocturne (Bull. Soc. ent. France [1899], p. 29). Son aire de dispersion est sans doute très étendue, comme celle de beaucoup d'espèces désertiques.

\title{
Gommunications.
}

\section{Description d'une espèce nouvelle de Rosalia de Formose}

[Col. Cerambycidae]

par P. BOPPE.

Rosalia Lesnei,n. sp. - Nigra, prothorace supra et infra elytrisque rubris; antennarum apud or et \& scapo pyriformi tenui et sat crebre punctato, articulis tertio, quarto et quinto apice valde peniciilatis, tertio, quarto, quinto, sexto et septimo apice dentatis; prothoracis disco utrinque macula rotunda nigra et in medio maculis duabus. Elytris élongatis et in singulo maculis quinque nigris, prima ad scutellum, in quarta anteriore parte maculis duabus, prima versus suturam, secunda versus latus, in disci medio singula macula transverse elongata (1) maculaque nigra in quarta posteriore parte. - Long. 26 mill., élytres 18 mill., larg. 6 mill.

Stature svelte. Antennes longues, dépassant les élytres chez le ơ de leurs quatre derniers articles, de leurs deux derniers seulement chez la \&, à scape noir, finement et densément ponctué, garni en dessous, ainsi que le $2^{\mathrm{e}}$ article, de longs cils soyeux, noirs; articles 3 , 4, 5 recouverts d'une pubescence noire couchée de laquelle émerge, surtout en dessous, de longs cils soyeux et noirs qui se condensent dans les deux sexes en une touffe épaisse à l'extrémité apicale de l'article

(1) Apud feminam nulla. 
Cette touffe cachant une épine relativement grêle mais longue et recourbée vers l'extrémité apicale; 6 et 7 dépourvus de cils mais possédant une épine apicale beaucoup plus faible, 8 à 10 inermes, 11 égal aux deux derniers. Tête noire, finement et densément ponctuée.

Prothorax rouge brique en dessus et en dessous, orné en dessus, sur la ligne médiane, de deux taches de pilosité noire, l'antérieure (la plus petite) presque ponctiforme, l'autre quadrangulaire près du bord postérieur; côtés tuberculeux, mais non épineux, ornés chacun d'une tache noire arrondie.

Scutellum noir, fortement sinué en arrière.

Saillies prosternale et mésosternale noires, également peu larges.

Élytres rouge brique, présentant une tache noire arrondie dans chacun des angles antéro-scutellaires. Le système de trois bandes noires habituel au genre Rosalia se retrouve ainsi modifié : la bande du quart antérieur est formée sur chaque élytre de deux taches arrondies, l'une sur la ligne médiane, l'autre sur le côté de l'élytre et très extérieurement.

La bande médiane est formée d'une tache allongée transversalement et s'étendant horizontalement jusqu'à 1 mill. des bords sutural et épipleural $\left({ }^{1}\right)$.

La bande postérieure se réduit à une assez grosse tache arrondie, sur la ligne médiane de l'élytre.

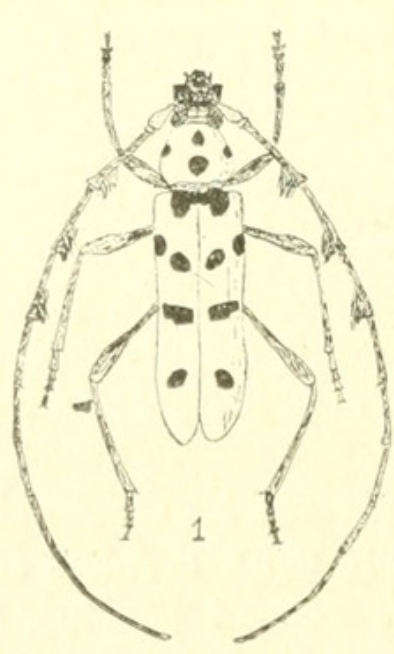

Fig. 1.

Rosalia Lesnei Boppe, $\overbrace{}^{x}$.

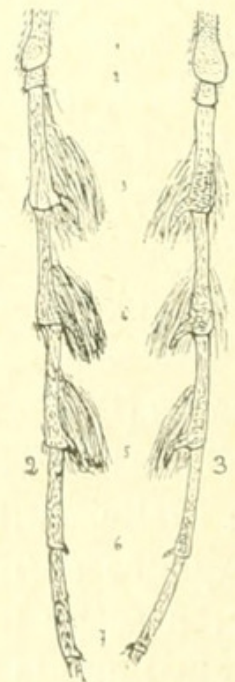

Fig. 2. - Antenne de la $Q$ de Rosalia Lesnei. - Fig. 3. - Antenne du $\sigma^{x}$.

(1) Cette tache, qui est de beaucoup la plus importante chez nos deux individus $\sigma^{x}$, fait totalement défaut chez notre unique $ᄋ$; il ne s'agit vraisemblablement ici que d'une variation individuelle. 
Abdomen et pattes entièrement noirs, fémurs assez nettement claviformes, tibias très allongés, $\mathbb{1}^{\mathrm{er}}$ article des tarses plus grand que 2 et 3 réunis.

Deux individus ot une o. Ma collection.

Patrie : Tai-Nan, Formose.

Cette espèce se place très naturellement entre le Rosalia Lameerei Brongniart et le Rosalia lateritia Hope, en tête par conséquent des Rosalia à livrée rouge (s.-g. Eurybatus Thoms.). Ses antennes à touffes de poils condensés à l'extrémité dans les deux sexes la rattachent en effet très intimement d'une part aux Rosalia à livrée bleue, dont $R$. Lameerei est une forme très caractérisée, d'autre part à $R$. lateritia, espèce à livrée rouge qui a perdu ce caractère chez le ỡ, mais l'a conservé chez la ㅇ.

Elle constitue un point de repère fort remarquable au point de vue de la distribution géographique des espèces du genre. Les espèces à livrée bleue, toutes confinées dans les régions tempérées de l'hémisphère boréal, ont comme caractère commun ces antennes à touffes condensées dans les deux sexes. Les espèces à livrée rouge, spéciales au contraire aux régions tropicales del'Asie orientale, ne présentent un caractère analogue que chez $\boldsymbol{R}$. lateritia et $\boldsymbol{R}$. Lesnei. Or $\boldsymbol{R}$. lateritia, dont, nous l'avons dit, les o ont seules ce caractère, présente une aire de dispersion assez étendue vers le nord, puisqu'elle est signalèe de Corée (1); R. Lesnei dont les deux sexes le possèdent aussi nettement que les espèces à livrée bleue, est originaire de Formose, d'une région intermédiaire par conséquent entre les deux zones fauniques du genre Rosalia.

Nous dédions cette espèce à M. P. Lesne, assistant au Muséum de Paris, en reconnaissance de son inépuisable obligeance.

M. Lameere, professeur à l'Université de Bruxelles, a publié ( $\left.{ }^{2}\right)$ en 1887 une monographie du genre Rosalia. Depuis cette époque cinq nouvelles espèces ont été décrites; elles peuvent se placer de la façon suivante dans le tableau ci-après, dont l'idée directrice est empruntée à celui de LAMEere :

I. Corps à pubescence bleue ou grise, ornée de taches ou de bandes noires.

Mandibules des ơ pourvues extérieurement d'une dent à la base (espèces européennes ou du nord de l'Asie).

(1) Lameere. Mon. du genre Rosalia, in Mém. Soc. ent. Belg., XXXI, p. 166.

(2) Lameere, $l$. $c$. 
Base des élytres granuleuses............. R. alpina L. Base des élytres sans granulation.

Épaules et mésosternum cendrés. Dent mandibulaire externe mousse. Prothorax tuberculé........... R. Batesi Harold.

Épaules et mésosternum noirs. Dent mandibulaire robuste, recourbée en dedans. Prothorax non tuberculé............. R. Lameerei Brongniart. Mandibules des $\vec{o}$ sans dent basale externe (espèce de l'Amérique du Nord)............. R. funebris Motsch.

II. Corps à pubescence jaune brunâtre chamois, orné de taches noires.................. R. Bouvieri Boppe.

III. Corps à pubescence vermillon.

Prosternum noir en dessous. Fémurs fusiformes ou linéaires.

Fémurs fusiformes. Antennes des + avec de forte houppes de poils; antennes des ơ à articles 3 -כ̆ fortement anguleux en dehors, mais non épineux.........

R. lateritia Hope.

Fémurs linéaires. Antennes des o sans houppe de poils, antennes des $\sigma^{x} \dot{a}$ art. 3-כ̈ munis d'une petite épine.

Élytres fortement dilatés en arrière. Antennes des 우

plus longues que le corps......... R. gravida Lam.

Élytres non dilatés en arrière. Antennes des o un

peu moins longues que le corps.. R. hariola Thoms.

Prosternum vermillon en dessous. Fémurs claviformes.

Abdomen entièrement noir.

Prosternum avec une tache noire, triangulaire en avant des hanches antérieures.... R. decempunctata Westw.

Prosternum sans tache noire en avant des hanches antérieures.

Extrémité apicale des élytres vermillon.

Antennes du ơ dépassant les élytres de leurs quatre derniers articles; les art. 3,4, כ̋ munis dans les deux sexes d'une touffeépaisse de poils condensés à l'extrémitė apicale.. R. Lesnei Boppe.

Antennes du ơ dépassant les élytres de leurs deux derniers articles; les art. $3, \mathbf{4}, \breve{\text { non mu- }}$ nis dans les deux sexes d'une épaisse touffe de 
poils condunsés à l'extrémité apicale. R. laeta Lam. Extrémité apicale des élytres noire sur le quart de

leur longueur. $\boldsymbol{R}$. borneensis Rothsch. et Jordan. Abdomen en grande partie vermillon.

Base des élytres vermillon.

Pronotum tuberculeux. $6^{\mathrm{e}}$ article antennaire non

épineux............. R. novempunctata Westw.

Pronotum non tuberculeux. $6^{\circ}$ article antennaire

épineux............. $R$. inexpectata Ritsema.

Base des élytres noires. Fémurs longuement clavi-

formes................ formosa Saunders.

\section{Notes brèves pour aider à la distinction des Longicornes du genre mallosia Muls. [CoL. Cerambidida] \\ par Maurice PIC.}

En montrant une boìte de sa collection, M. Maurice PIc indique brièvement quelques caractères faciles à voir pour aider à la distinction rapide de la plupart des espèces, races ou variétés qu'il a décrites dans le genre Mallosia Muls. (1) qui est un de ceux renfermant les plus belles espèces de la faune paléarctique.

M. costata Pic. - Se distingue, à première vue, par les élytres pourvus de côtes très marquées.

M. Delagrangei Pic. - Peut se distinguer, à première vue, de M. mirabilis Fald. et autres races, par les élytres dépourvus de macules blanches sur les côtés.

Parmi les variétés de $\boldsymbol{M}$. Gan glba ueri $\mathrm{Kr}$., la variété semirubra $\mathrm{Pic}$ se reconnaît à la coloration foncière plus ou moins roussâtre des élytres; la variété multimaculata Pic, en outre des macules peu irrégulières des élytres, se distingue facilement par la suture nettement et régulièrement pubescente.

M. brevipes Pic. - Est des plus distinctes, entre toutes les espèces, par ses pattes courtes, très poilues.

(1) Consulter, pour l'étude générale ou plus complète du genre Mallosia Muls., M. Pic, Mat. Longic. V, 2, 1905, p. 24 à 36; Mat. Long. VII, 1, 1908, p. 12 à 14 . 


\section{$2 \mathrm{BHL}$ Biodiversity Heritage Library}

Boppe, P. 1911. "Description d'une espèce nouvelle de Rosalia de Formose (Col., Cerambycidae)." Bulletin de la Société entomologique de France 1911, 103-107. https://doi.org/10.5962/bhl.part.2966.

View This Item Online: https://www.biodiversitylibrary.org/item/34144

DOI: https://doi.org/10.5962/bhl.part.2966

Permalink: https://www.biodiversitylibrary.org/partpdf/2966

\section{Holding Institution}

Smithsonian Libraries

\section{Sponsored by}

Smithsonian

\section{Copyright \& Reuse}

Copyright Status: NOT_IN_COPYRIGHT

This document was created from content at the Biodiversity Heritage Library, the world's largest open access digital library for biodiversity literature and archives. Visit BHL at https://www.biodiversitylibrary.org. 